N. Just*, O. Carpentier", C. Brzezinki ${ }^{\mp}$, F. Steenhouwer ${ }^{+}$and D. Staumont-Sallé

Depts of *Pneumology and Allergology, "Internal Medicine, ${ }^{+}$Pneumology, Victor Provo Medical Centre, Roubaix, and "Dept of Dermatology, Claude-Huriez Hospital, Lille University Medical Hospital, Lille, France.

Correspondence: N. Just, Service de Pneumologie, Hôpital Victor Provo, 11-17 Bd Lacordaire, F-59100 Roubaix Cedex, France. E-mail: nicolas.just@ch-roubaix.fr

Statement of Interest: None declared.

\section{REFERENCES}

1 Brockow K, Romano A, Blanca M, et al. General considerations for skin test procedures in the diagnosis of drug hypersensitivity. Allergy 2002; 57: 45-51.
2 Janier M, Froidevaux D, Lons-Danic D, et al. Acute generalized exanthematous pustulosis due to the combination of chloroquine and proguanil. Dermatology 1998; 196: 271.

3 Luong MS, Bessis D, Raison-Peyron N, et al. Severe mucocutaneous necrotizing vasculitis associated with the combination of chloroquine and proguanil. Acta Derm Venereol 2003; 83: 141.

4 Bocquet $\mathrm{H}$, Bagot M, Roujeau JC. Drug-induced pseudolymphoma and drug hypersensitivity syndrome (Drug Rash with Eosinophilia and Systemic Symptoms: DRESS). Semin Cutan Med Surg 1996; 15: 250-257.

5 Chen YC, Chiu HC, Chu CY. Drug reaction with eosinophilia and systemic symptoms: a retrospective study of 60 cases. Arch Dermatol 2010; 146: 1373-1379.

6 Kardaun SH, Sidoroff A, Valeyrie-Allanore L, et al. Variability in the clinical pattern of cutaneous side-effects of drugs with systemic symptoms: does a DRESS syndrome really exist? Br J Dermatol 2007; 156: 609-611.

7 Santiago F, Goncalo M, Vieira R, et al. Epicutaneous patch testing in drug hypersensitivity syndrome (DRESS). Contact Dermatitis 2010; 62: 47-53.

DOI: $10.1183 / 09031936.00123710$

\title{
A specific point-of-care screen for infectious pleural effusions using reagent strips
}

\section{To the Editors:}

The timely diagnosis of infectious pleural effusions may be challenging [1]. Infection should be suspected in patients with radiographic pleural opacities and fever. However, fever is often present not only in parapneumonics, but also in tuberculous and, less commonly, other exudative effusions. Pleural fluid analysis is the appropriate tool to aid in the differential diagnosis, but it may not be available on an emergency basis.

The dipstick leukocyte esterase test is intended to detect leukocytes in urine, but it has also been applied to other biological specimens for the rapid diagnosis of infection. The test uses the ability of the esterase enzyme present in the polymorphonuclear leukocytes of the sample to split heterocyclic carboxylates and form a pyrrole. The latter reacts with a diazonium salt producing a violet colour in the reagent strip. We hypothesised that testing pleural fluid with leukocyte esterase reagent strips may help to rule in or out a bacterial aetiology (mostly parapneumonic effusions) in just a few minutes. If this were the case, the test would be of interest to emergency departments, war-time military medical units and countries with limited medical facilities [2].

We prospectively evaluated all consecutive patients who underwent a diagnostic thoracentesis at the Arnau de Vilanova University Hospital (Lleida, Spain) from September 2009 to November 2010. The ethics committee approved the study and all patients gave written informed consent.

After the pleural tap, the fluid was sent for routine biochemical, microbiological and cytological analyses and immediately tested with Combi-Screen $₫$ (Analyticon Biotechnologies AG, Lichtenfels, Germany) reagent strips designed for urine, by an investigator blinded to the clinician diagnosis. Similarly, the clinicians were unaware of the dipstick results until the end of the study. A drop of non-centrifuged pleural fluid collected in heparinised tubes was applied to the leukocyte label of the reagent strip. Then the colour change was visually read against the colour scale on the container at precisely $2 \mathrm{~min}$. The results were recorded as: 0 (no change), $1+, 2+$ or $3+$. The finding of $1+$ or more was considered a positive test. The price of each strip was $€ 0.32$.

The aetiology of pleural effusions was established by standard clinical criteria [1]. Patients were divided into three groups, namely, nonmycobacterial infectious, tuberculous and noninfectious effusions. Complicated parapneumonic effusions refer to those non-purulent effusions associated with bacterial pneumonia which require a tube thoracostomy for cure. Empyema was defined as pus in the pleural space. Between-group comparisons of qualitative and quantitative variables were made by the Fischer exact test and Kruskal-Wallis test, respectively. The discriminative properties of reagent strips were evaluated using receiver operating characteristic curve analysis. We calculated measures of test efficacy, such as sensitivity, specificity and likelihood ratios (LR). If the $2 \times 2$ contingency contained any zeros, resulting in likelihood estimates of zero or infinity, 0.5 was added to all the counts for calculating the LR and the respective confidence intervals. The correlation between semi-quantitative dipstick results and the number of leukocytes and neutrophils in the pleural fluid was performed using the Spearman correlation coefficient. In a small subgroup of patients, weighted $\kappa$ was used to assess the level of agreement on dipstick readings.

Out of 145 patients recruited during the study period, 17 were excluded due to the presence of extremely bloody fluids, which make test strips uninterpretable, or because of an uncertain cause of the pleural effusion. The aetiological distribution of the final 128 pleural effusion patients, of whom 
85 were males and 43 females with a median (quartiles) age of 69 (50-81) yrs, were as follows: 42 infectious (34 parapneumonics and eight non-parapneumonics), 15 tuberculous and 71 noninfectious fluids. The pleural fluid culture was positive in six patients from the first group (three parapneumonics and three non-parapneumonic infections). Pleural fluid reagent strip analysis is presented in table 1 . The diagnostic performance of the leukocyte esterase for identifying nonmycobacterial infections against the remaining causes of pleural effusion was: sensitivity $42 \%$ (95\% CI $27-57 \%$ ); specificity $100 \%$ (95\% CI $97-$ $100 \%$ ); positive LR 74.8 (95\% CI 4.6-1212); negative LR 0.57 (95\% CI 0.44-0.74); and area under the curve 0.71 (95\% CI 0.61-0.82). In parapneumonic effusions, test sensitivity was unrelated to the infectious stage, whether simple, complicated or empyema. The positivity of reagent strips correlated with the number of neutrophils $(\mathrm{r}=0.591, \mathrm{p}<0.001)$ and leukocytes $(\mathrm{r}=0.584$, $\mathrm{p}<0.001)$ in the pleural fluid. Finally, the two-observer interrater agreement for the last 22 dipstick readings produced a $\mathrm{K}$ statistic of $0.95(0.89-0.99, \mathrm{p}<0.001)$.

It was found that leukocyte esterase activity testing by dipstick may help rule in a nonmycobacterial infectious effusion due to its high specificity. The overall discriminatory properties of this simple and cheap test compare favourably with those of some biomarkers of pleural infection in use, such as C-reactive protein [1]. Only two previous studies have evaluated the ability of urine test strips when applied to pleural fluid specimens for identifying infectious effusions [3, 4]. However, they were performed on very selected populations. In the first study, AzOULAy et al. [3] tested commercially available reagent strips (Multistix $8 \mathrm{SG}_{\mathbb{R}}$; Bayer, Leverkusen, Germany) on 78 patients who had been admitted to an intensive care unit, including 35 with infectious effusions, 23 with noninfectious exudates and 20 transudates. A leukocyte esterase grade $\geqslant 2$ on a six-grade scale, read by a colorimeter, displayed a sensitivity of $42 \%$, specificity of $91 \%$, positive LR of 4.9 and negative LR of 0.63 for the detection of infectious effusions among exudates. Nevertheless, the infectious group was a mixture of two tuberculous pleuritis, 21 parapneumonic and 14 empyema patients. Most importantly, 12 out of the 15 patients with infectious effusions and a leukocyte esterase test result grade of $\geqslant 2$ had empyemas (defined as a positive pleural fluid culture). The plausible purulent appearance of most of these empyemas would have made the diagnosis clear without the use of reagent strips. In the second study, CASTELLOTE et al. [4] evaluated the performance of a reagent strip for leukocyte esterase (Aution ${ }^{\circledR}$; Menarini Diagnostics, Firenze, Italy) to diagnose spontaneous bacterial pleuritis in 47 pleural fluid samples from patients with hepatic hydrothorax. When a test result cut-off of $\geqslant 2$ on a five-grade scale was considered positive, reagent strips exhibited the following accuracy: sensitivity $91 \%$, specificity $80 \%$, positive LR 4.58 and negative LR 0.10 . However, if the cut-off was set at $\geqslant 3$, these figures were $83 \%, 100 \%, 58.1$ and 0.17 , respectively. Our study extends the applicability of dipstick tests to all patients with pleural effusion admitted to a general ward and notably reveals the negativity of reagent strips in pleural tuberculosis.

This study should be interpreted in the light of certain limitations. First, we used a visual reading rather than a colorimeter to make dipsticks more clinically applicable at the expense of a potentially less accurate interpretation. Extremely bloody fluids $\left(>50,000\right.$ red blood cells $\left.\cdot \mu \mathrm{L}^{-1}\right)$, an appearance which is present in $14 \%$ of 423 parapneumonics and $4 \%$ of 239 tuberculous effusions according to our unpublished database, preclude adequate recognition of colour field changes. Secondly, reagent strips did not discriminate between complicated and uncomplicated parapneumonic effusion, which is a more clinically relevant concern than the infectious or noninfectious differentiation. Thirdly, it seems unlikely that this test would be able to separate parapneumonic effusions from other neutrophilrich effusions, including the $10 \%$ of tuberculous pleuritis which have this characteristic [5]. Because of the absence of such a comparator group in our series, further confirmatory studies are needed. Finally, it should be stressed that reagent strips should not be viewed as a substitute for biochemical analysis.

To conclude, reagent strips may speed up the bedside diagnosis of infectious effusions. As the value of a diagnostic test lies in its ability to distinguish between otherwise commonly confused disorders, it is thought by the authors that the main

\begin{tabular}{|c|c|c|c|c|c|c|c|}
\hline \multirow[t]{2}{*}{ Aetiology } & \multirow[t]{2}{*}{ Subjects } & \multirow{2}{*}{$\begin{array}{l}\text { Pleural fluid leukocyte } \\
\text { count cells } \mu^{-1}\end{array}$} & \multirow{2}{*}{$\begin{array}{l}\text { Pleural fluid neutrophil } \\
\text { count cells } \mu^{-1} \mathrm{~L}^{-1}\end{array}$} & \multicolumn{4}{|c|}{ Reagent strip ${ }^{\#}$} \\
\hline & & & & 0 & $1+$ & $2+$ & $3+$ \\
\hline Infectious (nonmycobacterial) & $42(32.8)$ & $2730(900-8615)$ & $1096(285-6862)^{\S}$ & $24(55.8)$ & 4 & 9 & 5 \\
\hline Parapneumonics & 34 & $3100(780-10950)$ & $2646(808-9724)$ & 20 & 2 & 8 & 4 \\
\hline Empyema & 4 & $21136(2878-113950)$ & 19022 (10145-19022) & 0 & 0 & 1 & 3 \\
\hline Non-associated pneumonic processes & 8 & $3850(1405-20855)$ & $2716(531-6020)$ & 4 & 2 & 1 & 1 \\
\hline Tuberculosis & $15(11.7)$ & $2000(1440-3600)$ & $152(69-597)$ & $15(100)$ & 0 & 0 & 0 \\
\hline Noninfectious $^{+}$ & $71(55.5)$ & $650(260-1600)_{f}$ & $180(30-438)$ & $71(100)$ & 0 & 0 & 0 \\
\hline Total & 128 & $1000(403-3500)$ & $336(85-1575)$ & $110(86)$ & 4 & 9 & 5 \\
\hline
\end{tabular}

Data are presented as $n(\%)$, median (quartiles) or $n .{ }^{\#}:$ higher frequency of positive reagent strips in the infectious group compared with other groups ( $p<0.001$ by Fisher exact test). $\because$ : subdiaphragmatic infection $(n=4)$, oesophageal perforation $(n=2)$, penetrating trauma $(n=1)$ and spontaneous bacterial empyema $(n=1),{ }^{+}$: malignancy $(n=26)$, heart failure $(n=21)$, hepatic hydrothorax $(n=6)$, other transudates $(n=4)$, post-abdominal surgery $(n=4)$, pulmonary embolism $(n=2)$, blunt trauma $(n=2)$, viral pleuropericarditis $(n=3)$, systemic lupus erythematosus $(n=1)$, acute pancreatitis $(n=1)$ and benign asbestos pleural effusion $(n=1){ }^{s}$ : significantly higher than the respective values in other groups by Kruskal-Wallis test $(p<0.001) .{ }^{f}$ : significantly lower than the respective values in other groups by Kruskal-Wallis test $(p<0.001)$. 
application for this use of dipsticks is bacterial-mycobacterial effusion discrimination, particularly in the setting of resourcelimited healthcare systems. However, where access to standard biochemical pleural fluid analysis, including adenosine deaminase [5], is available, dipstick tests add little value to current practice.

\section{J.M. Porcel*, A. Esquerda" ${ }^{*}$ and S. Bielsa*}

Depts of *Internal Medicine and "Laboratory Medicine, Pleural Diseases Unit, Arnau de Vilanova University Hospital, Unitat de Recerca Biomèdica de Lleida, Lleida, Spain.

Correspondence: J.M. Porcel, Dept of Internal Medicine, Arnau de Vilanova University Hospital, Avda Alcalde Rovira Roure 80, 25198 Lleida, Spain. E-mail: jporcelp@yahoo.es

\section{REFERENCES}

1 Porcel JM, Vives M, Cao G, et al. Biomarkers of infection for the differential diagnosis of pleural effusions. Eur Respir J 2009; 34: 1383-1389.

2 Peeling RW, Mabey D. Point-of-care tests for diagnosing infections in the developing world. Clin Microbiol Infect 2010; 16: 1062-1069.

3 Azoulay E, Fartoukh M, Galliot R, et al. Rapid diagnosis of infectious pleural effusions by use of reagent strips. Clin Infect Dis 2000; 31: 914-919.

4 Castellote J, Lopez C, Gornals J, et al. Use of reagent strips for the rapid diagnosis of spontaneous bacterial empyema. J Clin Gastroenterol 2005; 39: 278-281.

5 Porcel JM, Esquerda A, Bielsa S. Diagnostic performance of adenosine deaminase activity in pleural fluid: a single-center experience with over 2100 consecutive patients. Eur J Intern Med 2010; 21: 419-423.

\section{Response of automatic continuous positive airway pressure devices in a normal subject}

\section{To the Editors:}

Obstructive sleep apnoea (OSA) is a prevalent disease whereby people become unable to breathe when asleep. During sleep the pharyngeal walls are sucked in and collapse completely, causing full (termed apnoea) or incomplete (termed hypopnoea) abolition of airflow. OSA can lead to daytime fatigue and sleepiness, impairment in cognitive functions, motor vehicle crashes, hypertension, cardiovascular disease, stroke and premature deaths [1].

OSA is easily treated with continuous positive pressure applied throughout sleep [2]. The right amount of pressure is determined individually during a titration night, when pressure is gradually increased to keep the airway open to allow for normal sleep and breathing [2]. In 1993 it was postulated that this process of titration could be performed with a stand alone blower equipped with a microprocessor guiding the positive pressure [3]. The idea was to use the blower as a monitoring device identifying abnormal breathing events and react by increasing pressure until disappearance of the abnormal events. The device would then slowly decrease pressure until events reappeared and the pressure would then be increased again [3]. These machines, termed automatic continuous positive airway pressure devices (autoCPAP), were proposed as monitoring or even diagnostic machines [4], as devices used for one night to determine the fixed CPAP level to be used thereafter [5] or even as therapeutic machines to be used every night and to adapt the pressure necessary to keep the pharynx open at all times [6].

When autoCPAP devices were tested in a bench study it was shown that no two devices reacted alike, that many devices did not even recognise the events they were supposed to act upon and that even when recognising the events, many were not able to correct them [7].

Contrary to pharmaceutical treatments, autoCPAP devices have never been submitted to phase I studies. In other words, they have never been applied to normal subjects [8]. If the assumptions behind these devices are correct, an autoCPAP applied to a normal subject should detect no abnormality in breathing and therefore its positive pressure should remain at the minimum level $\left(4 \mathrm{cmH}_{2} \mathrm{O}\right.$ in general) necessary to wash out the expired $\mathrm{CO}_{2}$ from the mask. We decided to test this scenario in a double-blind experiment in one normal volunteer.

The volunteer subject was a 29-yr-old never-smoker male with an unremarkable medical history. On medical examination weight was $90.5 \mathrm{~kg}$ and height was $186 \mathrm{~cm}$. Blood pressure was $115 / 75 \mathrm{mmHg}$, heart rate was 63 beats $\cdot \mathrm{min}^{-1}$ and the transcutaneous $\mathrm{O}_{2}$ saturation was $98 \%$. The rest of the physical examination was unremarkable. The Mallampati score was 1.

The subject signed an informed consent form and received a payment of $€ 100$ for each polysomnography (PSG). On two successive nights PSG was recorded. After verifying the normality of sleep and breathing he was given a CPAP machine (Good-Knight 420G device ${ }^{\circledR}$; Covidien, Mansfield, MA, USA) with mask (Flexy Fit407®; Fisher and Paykel, Auckland, New Zealand) and gears to use at home at a pressure of $4 \mathrm{cmH}_{2} \mathrm{O}$. After a few nights habituation, a new PSG was performed. After verifying that the patient could sleep normally with the minimum pressure CPAP the experimental study was started. 